\title{
Making Giant Unilamellar Vesicles via Hydration of a Lipid Film
}

\author{
Suliana Manley ${ }^{1}$ and Vernita D. Gordon ${ }^{2}$ \\ ${ }^{1}$ National Institutes of Health, Cell Biology and Metabolism Branch, Bethesda, Maryland \\ ${ }^{2}$ University of Illinois Urbana-Champaign, Department of Materials Science and \\ Engineering, Urbana, Illinois
}

\begin{abstract}
This unit describes protocols for making giant unilamellar vesicles (GUVs) based on rehydration of dried lipid films. These model membranes are useful for determining the impact of membrane and membrane-binding components on lipid bilayer stiffness and phase behavior. Due to their large size, they are especially amenable to studies using fluorescence and light microscopy, and may also be manipulated for mechanical measurements with optical traps or micropipets. In addition to their use in encapsulation, GUVs have proven to be useful model systems for studying many cellular processes, including tubulation, budding, and fusion, as well as peptide insertion. The introduction of enzymes or proteins can result in reorganization, leading to such diverse behavior as vesicle aggregation, fusion, and fission. Curr. Protoc. Cell Biol. 40:24.3.1-24.3.13. (C) 2008 by John Wiley \& Sons, Inc.
\end{abstract}

Keywords: liposomes $\bullet$ giant vesicles $\bullet$ electroformation $\bullet$ liposome swelling • model membranes

\section{INTRODUCTION}

This unit describes protocols for forming giant unilamellar vesicles (GUVs). The techniques described use hydration of dried lipid films, either by electroformation (Angelova et al., 1992) or by gentle hydration (Mueller et al., 1983). Swelling by electroformation (see Basic Protocol 1 and Alternate Protocol 1) produces a high yield of GUVs, typically 10 to $100 \mu \mathrm{m}$ in diameter. Electroformation is not recommended for use with buffered solutions or charged lipids; instead, gentle hydration should be used (see Basic Protocol 2 and Alternate Protocols 2 and 3). An alternative technique that uses rapid evaporation is not described here (Moscho et al., 1996). Because nonfunctionalized vesicles are generally only weakly adhesive to many substrates, they may drift during imaging. Alternate Protocols 1 and 3 produce GUVs that are attached to wires or glass, thereby eliminating drift.

\section{PREPARING GUVS BY ELECTROFORMATION ON INDIUM TIN OXIDE-COATED PLATES}

This protocol is ideal for producing several milliliters of GUVs at $\sim 10^{3}$ to $10^{4}$ per $\mu \mathrm{l}$, which can then be submitted to a variety of different conditions. If desired, indium tin oxide (ITO) plates of coverslip thickness may be purchased, thus allowing observation of the growth of GUVs. For microscopy, lipids are generally rehydrated in aqueous sucrose solutions. Resuspension in equimolar glucose results in vesicles containing an interior solution that is denser than the suspending medium. This is commonly used in inverted microscopy to force vesicles to sink to the bottom of the sample chamber, next to the objective; switching solutions (glucose interior, sucrose exterior) causes vesicles to cream to the top for observation by upright microscopy. This also allows for application of osmotic stress and deflation using different molarities of sucrose and glucose. Furthermore, if fluorescent lipids are not incorporated, the difference in index of

Current Protocols in Cell Biology 24.3.1-24.3.13, September 2008

Published online September 2008 in Wiley Interscience (www.interscience.wiley.com).

DOI: $10.1002 / 0471143030 . c b 2403 s 40$

Copyright $(2008$ John Wiley \& Sons, Inc.
UNIT 24.3

BASIC

PROTOCOL 1

Lipids

24.3.1

Supplement 40 
refraction produced by the sugar solutions allows vesicles to be viewed using differential interference contrast or phase-contrast microscopy.

\section{Materials}

$100 \mu \mathrm{l}$ lipid in chloroform, at 5 to $10 \mathrm{mg} / \mathrm{ml}$ (see Support Protocol 1)

Up to $600 \mathrm{mM}$ sucrose, or sterile deionized $\mathrm{H}_{2} \mathrm{O}$

25-cm² ITO-coated plates (e.g., Delta Technologies, Ltd., part no. CG-511IN50x50/1.1; http://www.delta-technologies.com/)

Sealed chamber (e.g., vacuum desiccator) connected to dry nitrogen or vacuum source

Vacuum grease

Teflon holder and spacers for ITO-coated plates (see Support Protocol 2)

Oven with hole to introduce electrodes or heating bath (for lipid systems in which at least one component has a chain-melting transition temperature, $T_{\mathrm{m}}$, above room temperature)

Electrodes ("Mini-Plunger to BNC Male" or equivalent; e.g., Radio Shack)

Function generator with readout for voltage and frequency (Stanford Research Systems, Ltd., http://www.thinksrs.com/)

\section{Prepare dry lipid film on ITO plates}

1. Prepare lipid mixtures (see Support Protocol 1).

2. Using a glass Pasteur pipet, deposit 2 to 3 drops of lipid mixture $(\sim 25 \mu \mathrm{l})$ onto the conductive side of a $25-\mathrm{cm}^{2}$ ITO-coated plate.

Less lipid should be used for a smaller plate; maintain coverage of $\sim 1 \mu \mathrm{l} / \mathrm{cm}^{2}$.

3. Before the solution dries, use the side of the pipet to quickly spread the lipid over the surface of the plate using a single swipe. Repeat with second plate.

Viewed in reflection, the lipid film should appear as colored interference fringes. The film should be as uniform as possible. Nonuniform films may result in a larger number of multilamellar or small vesicles. See Commentary for more information on the influence of film properties.

4. Place plates under vacuum at room temperature for at least $2 \mathrm{hr}$ and up to overnight, until ready for use.

\section{Carry out electroformation}

5. Assemble plates into parallel assembly in the Teflon holder, separated by spacers.

To prevent leakage, a small amount of vacuum grease may be spread around any seams in the Teflon holder. Parafilm or plastic wrap may be used to secure the assembly and render it leakproof.

6. Fill assembly with up to $600 \mathrm{mM}$ sucrose solution or sterile deionized water.

Choice of solvent and concentration is to be determined by the amount of osmotic stress, density, and index mismatch required for the experiment, and is a tunable parameter that is purely up to the experimenter.

7. Optional: For lipid mixtures or lipids with low $T_{\mathrm{m}}$, place the assembly in an oven or heating bath set above $T_{\mathrm{m}}$ for the highest-melting component.

For most unsaturated lipids and for many saturated lipids with tails up to 16 carbon atoms long, $50^{\circ} \mathrm{C}$ is sufficient.

Making Giant Unilamellar Vesicles via

Hydration of a Lipid Film

24.3.2

8. Attach one electrode to each plate.

Chamber should be covered to eliminate evaporation.

9. Set the function generator to output AC current (a sine wave), with amplitude $2 \mathrm{~V}$, frequency $10 \mathrm{~Hz}$. Incubate 1 to $2.5 \mathrm{hr}$. 
10. Remove assembly from function generator and oven. Use a pipet or syringe needle to extract vesicles. Take care to minimize shear stresses by using a large pipet-tip opening or syringe needle and slow extraction to maximize the number of vesicles that survive this transfer.

If using membrane dyes, unilamellar vesicles can be identified under the microscope as vesicles with the lowest fluorescence from the membrane.

11. Use GUVs within a few hours of formation (although a fraction will survive overnight).

12. Store GUVs at room temperature.

\section{MAKING LIPID MIXTURES}

This protocol describes the proper handling and storage of lipids, and gives some guidelines for selecting lipid mixtures.

\section{Choice of lipids}

Choosing a lipid composition is the first step in planning your experiments with GUVs. For testing your ability to make GUVs, a single, unsaturated lipid such as dioleoylphosphatidylcholine (DOPC) may be useful for troubleshooting. For model raft mixtures, a 1:1:1 molar composition of DOPC:sphingomyelin:cholesterol is typical, and is commonly referred to as the canonical raft mixture. However, this mixture produces GUVs that may not be phase-separated at room temperature. Therefore, if your microscope does not have the capability to be cooled, a mixture with a lower level of cholesterol that will readily phase-separate at room temperature may be preferable. For phase diagrams that provide the lipid phase melting temperatures of a range of ternary mixtures (Veatch and Keller, 2002), the influence of aliphatic chain length (Veatch and Keller, 2003) and sterol type (Bacia et al., 2005; Beattie et al., 2005) have also been investigated.

Lipid mixtures for peptide- and protein-binding experiments must be chosen carefully, since binding may depend strongly on lipid composition. In particular, it may be necessary to incorporate charged lipids in order to achieve binding (Bigay et al., 2003). A reasonable choice of lipids may be dictated by the natural lipid environment of the protein of interest. Biotinylated lipids are also available, and allow binding of vesicles to streptavidin or streptavidin-treated surfaces and objects.

Fluorescent lipid probes are useful for imaging vesicles and for determining phase behavior (Bagatolli, 2006). Headgroup-labeled phospholipids are typically used for these purposes, since the bulky fluorophore may interfere with lipid ordering if attached to an aliphatic chain. Several probes are available with readily accessible excitation wavelengths, including rhodamine-, Texas Red-, NBD- and fluorescein-conjugated lipids. These probes all partition preferentially into the liquid-disordered, non-raftlike phase, and are typically added at concentrations of $\sim 0.1 \mathrm{~mol} \%$. Probes that partition into the liquid-ordered, raftlike phase can also be desirable for probing curvature and mobility; these include perylene (Baumgart et al., 2003) and cholera toxin B binding to GM1 gangliosides (Bacia et al., 2005). The lipid probe Laurdan is a particularly powerful tool for studying local membrane ordering; the spectrum of the Laurdan molecule is dependent on the polarity of its surroundings and therefore reflects the packing density of neighboring lipids (Bagatolli and Gratton, 2000). By measuring the spatially resolved spectra, a mapping of phases can be derived. However, Laurdan requires two-photon excitation, due to its low excitation wavelength and its rapid photobleaching.
SUPPORT

PROTOCOL 1

Lipids

24.3.3

Supplement 40 


\section{Materials}

Lipid (Avanti Polar Lipids; when received, store at $-20^{\circ} \mathrm{C}$ or lower)

Chloroform

Methanol (optional)

Argon or nitrogen gas

Glass vials with Teflon closures

Microdispensers with glass bores (Drummond), or Hamilton syringes

Teflon tape

1. Rehydrate lipids supplied as powder in chloroform or a 2:1 (v/v) mixture of chloroform:methanol.

See Commentary for more information on the influence of film properties.

2. Handle lipids in chloroform with glass, stainless steel, or Teflon labware. Store all solutions in glass vials with Teflon-lined caps.

3. Use microdispensers with glass bores or Hamilton syringes to measure volumes for making lipid mixtures.

Typically, mixtures should be made in small volumes $(\sim 100 \mu l)$ or stored as aliquots to avoid contamination from handling.

4. Rinse all surfaces to come into contact with lipid solutions with chloroform.

This includes microdispenser tips, glass bores, glass vials, ITO-coated plates, platinum wires, and Teflon plates.

5. After use, store solutions under argon or nitrogen gas at $-20^{\circ} \mathrm{C}$ or lower to prevent hydrolysis or oxidation of lipids. After sealing, wrap caps in Teflon tape.

SUPPORT PROTOCOL 2

Making Giant Unilamellar Vesicles via

Hydration of a Lipid Film

\subsection{4}

\section{DESIGN OF A PTFE (TEFLON) HOLDER FOR ELECTROFORMATION ON ITO-COATED PLATES}

This protocol describes the design of a Teflon holder for ITO-coated plates used in electroformation. The dimensions should be adjusted to accommodate the size of the plates. A polytetrafluoroethene (PTFE, or Teflon) block is machined to prepare the holder. The holder consists of two pieces of Teflon designed to fit together. The design considerations are that plates should be held 2 to $5 \mathrm{~mm}$ apart. The inner dimensions of each Teflon piece should slightly exceed (by $\sim 1 \mathrm{~mm}$ ) those of the ITO-coated plates. An example is shown in Figure 24.3.1, for plates with dimensions $50 \times 50 \mathrm{~mm}$.

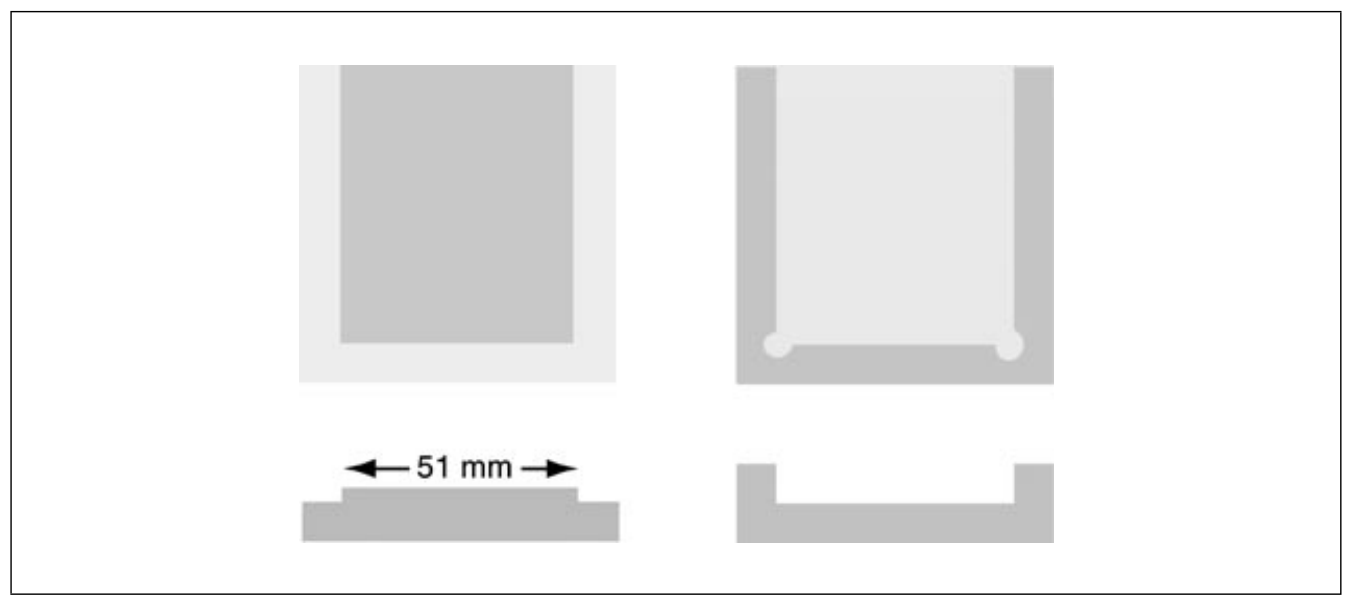

Figure 24.3.1 Teflon holder for ITO-coated plates, made from two pieces (shown left and right), front view and side view (shown top and bottom). 
PREPARING GUVS BY ELECTROFORMATION ON PLATINUM WIRES

This protocol produces a high yield of GUVs in a sample-chamber geometry that is well adapted for viewing under a microscope. Vesicles adhere to the platinum wire electrodes on which they form, so they remain largely stationary, without optically resolvable thermal fluctuations below $\sim 45^{\circ} \mathrm{C}$. If desired, the electroformation chamber can be adopted to allow harvesting of vesicles for experimentation on individual, isolated GUVs by using hydrodynamic flow along and across the electrodes.

Additional Materials (also see Basic Protocol 1)

5 to $10 \mu$ l of lipid solution in chloroform (Support Protocol 1) at concentration of 0.5 to $0.66 \mathrm{mM}(\sim 0.5 \mathrm{mg} / \mathrm{ml})$

Up to $600 \mathrm{mM}$ sucrose, or sterile deionized $\mathrm{H}_{2} \mathrm{O}$

Platinum wires, 0.5 - to $2.0-\mathrm{mm}$ diameter

Microdispensers with glass bores (Drummond), or Hamilton syringes

Sealed chamber (e.g., vacuum desiccator) connected to dry nitrogen or vacuum source

Electroformation chamber (see Support Protocol 3), assembled with platinum wires

\section{Prepare lipid-coated platinum wires}

1. If using more than one species of lipid, mix in the desired ratio (see Support Protocol 1) and dilute in chloroform. Add membrane dye(s) and other components to be incorporated into the membrane, if desired, so that the total concentration of membrane components in chloroform solution remains $\sim 0.5$ to $0.66 \mathrm{mM}$, or $\sim 0.5 \mathrm{mg} / \mathrm{ml}$.

2. Using a glass microdispenser or Hamilton syringe, deposit solution onto platinum wires in drops of $\sim 1 \mu \mathrm{l}$, so that drops are pendant from the wires.

Drops can be closely spaced along the wires, but care should be taken that they do not coalesce; too high a concentration of lipid will result in multilamellar vesicles.

3. Dry lipid onto wires in nitrogen environment or under vacuum for $2 \mathrm{hr}$ to overnight at room temperature.

\section{Electroform GUVS}

4. Add water (or other aqueous solution) to the chamber so that the wires are covered.

Choice of solvent and concentration is to be determined by the amount of osmotic stress, density, and index mismatch required for the experiment, and is a tunable parameter that is purely up to the experimenter.

5. If necessary, heat chamber so that the temperature is above $T_{\mathrm{m}}$ for the highest-melting component in the system.

For lipid systems in which $T_{m}$ for one or more component is above room temperature, the electroformation chamber must be heated above $T_{m}$ for the highest-melting component in the system. This may be done using a heating bath, by heating the chamber directly using resistive heating or Peltier plates, or by preheating the hydration solution, if the duration of electroformation is less than the time for the solution temperature to drop below $T_{m}$.

6. Apply a 2- to $10-\mathrm{V}, 10-\mathrm{Hz}$ AC electrical field across the electrodes for 30 to $120 \mathrm{~min}$.

During electroformation, care should be taken that the hydrating solution is in continuous contact with the electrode wires. If necessary, additional hydrating solution can be added to compensate for evaporation, but care should be taken to minimize hydrodynamic disturbances in the chamber, because these will wash vesicles off electrodes.

Chambers can also be covered with a lid to reduce evaporation. 
Time for electroformation varies with the lipids used, as does the yield. In general, increasing the percentage of phosphatidylcholine (PC) lipids increases yield and lowers electroformation time. Most vesicles formed using this technique will be unilamellar. Multilamellar vesicles can be identified, if membrane dyes are used, by higher levels of fluorescence.

SUPPORT PROTOCOL 3

\section{DESIGN OF A CHAMBER FOR ELECTROFORMATION ON PLATINUM WIRES}

This protocol describes the design and construction of a chamber for electroformation on platinum wires, intended for stringent temperature control and to allow for harvesting of GUVs. This design can be adapted depending on the need for viewing and manipulating GUVs.

\section{Materials}

Platinum wires, 0.5 - to $2.0-\mathrm{mm}$ diameter

Aluminum block (machined; typical dimensions, $10 \times 4 \times 1 \mathrm{~cm}$ )

Electrical block fittings or terminal block fittings (McMaster-Carr, http://www.mcmaster.com)

Optional items:

PTFE seals

Cover glass for microscopy

Norland optical adhesive

Resistive heating wire

Ceramic insulators and fittings for electrical attachment

Thermocouple

\section{Chamber construction}

The essential elements of this electroformation chamber are provided by two platinum wires, which serve as the electrode substrates for formation of GUVs, and the aluminum block, machined to provide a water-tight chamber. If intended for viewing under an inverted microscope, this chamber may be created by machining a hole $\sim 2$ to $3 \mathrm{~cm}$ in diameter through the aluminum block and sealing a cover glass across the bottom using an epoxy that is nonreactive in water. Platinum-wire electrodes should be threaded through holes drilled in the side walls of this chamber so that they are parallel and spaced about $3 \mathrm{~mm}$ apart. These holes should be lined with PTFE or some other material so that the wires are electrically isolated from the aluminum block, and sealed so that the chamber is watertight using nonreactive adhesive and/or tightly packed PTFE tape. If viewing under an inverted microscope is intended, wire electrodes should be placed as close as possible to the bottom of the chamber, so that they are within the working distance of the microscope objective to be used. Electrical block fittings attached to the ends of the wires (which should protrude $\sim 1 \mathrm{~cm}$ outside the aluminum block) allow these electrodes to be coupled to a function generator for electroformation. This design is illustrated by the central chamber of Figure 24.3.2.

If this chamber is to be used to electroform vesicles in which one or more lipid component(s) has $T_{\mathrm{m}}$ higher than room temperature, the chamber can be constructed so that it is resistively heated. This can be done by drilling two parallel holes in the aluminum block and threading heating wire through them so that the water chamber is approximately circumscribed. If this or another heating method is used, the chamber should be constructed with a thermocouple positioned as closely as possible to the wire electrodes, so that the temperature can be monitored.

aking Giant Unilamellar Vesicles via

Working at elevated temperatures will cause more rapid evaporation of the aqueous solution. If the solution level drops below the wire electrodes, vesicles will be destroyed by contact with air. Furthermore, if solutions other than pure water are used, evaporation 


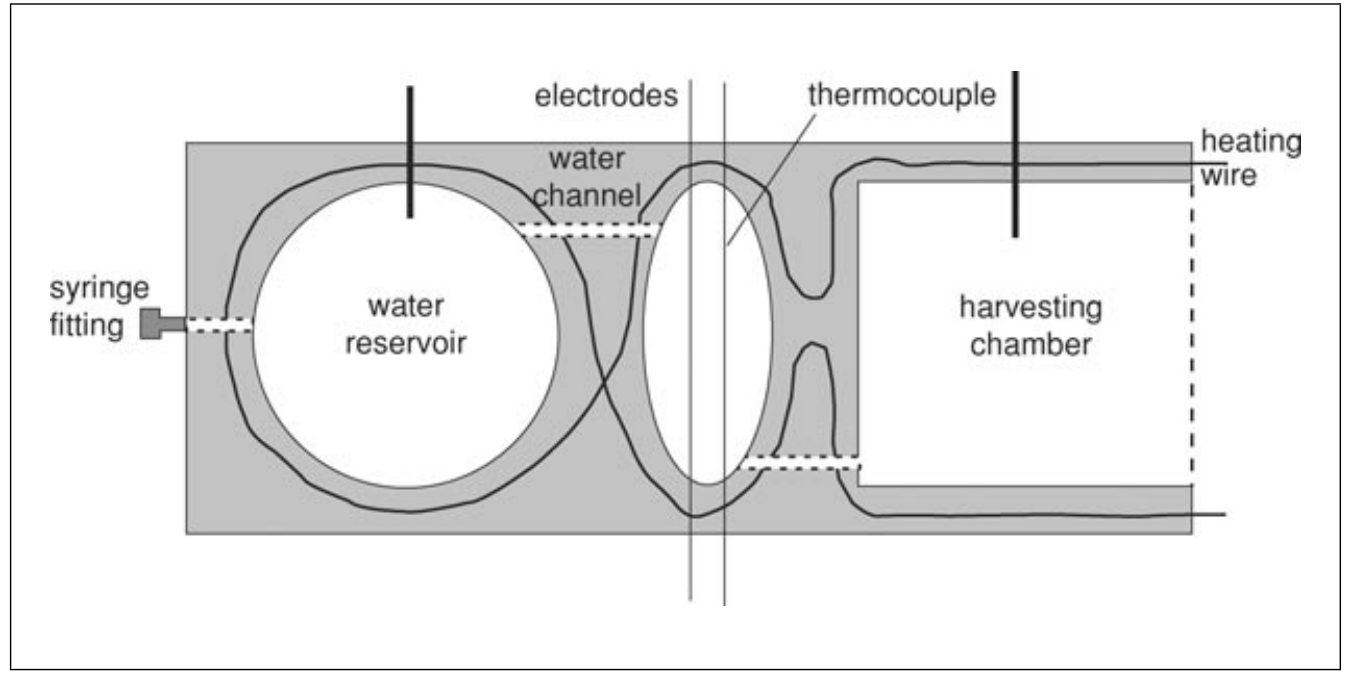

Figure 24.3.2 Electroformation assembly for platinum wires, showing optional water reservoir and harvesting chamber. The central chamber is essential, and contains the electrodes where the electroformation takes place.

will increase the concentration of solutes external to vesicle membranes (if any) and may cause membranes to become flaccid. If preventing this is desired, the chamber may be fitted with a sealed lid to reduce evaporation. However, such a lid may hinder application of some imaging techniques (if a lidded chamber is used under laser-scanning confocal microscopy, for example, reflection off the lid can significantly increase photobleaching throughout the sample). The effects of evaporation may also be mitigated by drilling the aluminum block to create a second water reservoir that is connected by a channel to the chamber in which electroformation and viewing are done. This is illustrated by the left chamber in Figure 24.3.2, marked "water reservoir." If this channel is higher (or lower) than the wire electrodes, water may be gently added to the reservoir to refill the electroformation chamber without significantly disrupting the lipids on the wires.

Alternatively, hydrodynamic flow can be deliberately used to flush GUVs off the wires so that isolated GUVs can be studied individually. Membranes should be fluid (above $T_{\mathrm{m}}$ ) for this to be effective. To avoid temperature-shocking vesicles, it may be desirable to preheat the water used for flushing so that it is at approximately the same temperature as the solution surrounding the membranes. An electroformation chamber that has been used to form and harvest GUVs using this method is sketched below, in Figure 24.3.2. The chamber is lidded and sealed except for the harvesting chamber, which is open on one end to allow access to vesicles. Dimensions depicted are not necessarily to scale.

\section{PREPARING GUVS BY SWELLING OFF OF PTFE (TEFLON)}

While electroformation is the most successful protocol to date for producing a high yield of giant unilamellar vesicles, it works well only for a limited range of lipid compositions, notably those high in phosphatidylcholine (PC) lipids (>25\% to $50 \mathrm{~mol} \%$ ) and low in charged lipids $(<10 \mathrm{~mol} \%)$, such as phosphatidylserine (PS) and phosphatidylglycerol (PG). Electroformation is also limited in the swelling solutions (buffers) to which it can be applied, because if salts are in the aqueous solution, the electric field can cause gas to be liberated by hydrolysis and form bubbles at the electrodes, where they destroy the vesicles. There is some evidence that electroformation can produce damaged membranes, and using a swelling technique to check the results seen for electroformed membranes allows the identification of artifacts (Gordon et al., in preparation). 
This swelling technique requires at least $\sim 10 \mathrm{~mol} \%$ charged lipids to produce unilamellar vesicles (the threshold amount varies with the other lipids in the system and with the buffer used). This technique can produce GUVs in standard, salt-containing biological buffers.

\section{Materials}

$20 \mathrm{mg} / \mathrm{ml} \mathrm{lipid} \mathrm{in} \mathrm{chloroform} \mathrm{(Support} \mathrm{Protocol} \mathrm{1)}$

Chloroform

Nitrogen gas

Swelling solution: $100 \mathrm{mM}$ sucrose or glucose prepared using sterile deionized $\mathrm{H}_{2} \mathrm{O}$

Microdispensers with glass bores (Drummond), or Hamilton syringes

PTFE (Teflon) sheet, $\sim 2 \times 2-\mathrm{cm}$ square, roughened with fine-grain sandpaper

(McMaster-Carr, http://www.mcmaster.com)

Desiccator chamber, attached to vacuum pump

Glass beaker

Incubator or oven

\section{Prepare dry lipid film}

1. If using more than one species of lipid, mix in the desired ratio as in Support Protocol 1. Add membrane dye(s) and other components to be incorporated into the membrane, if desired, so that the total concentration of membrane components in chloroform solution remains approximately $20 \mathrm{mg} / \mathrm{ml}$.

2. Using a glass microdispenser or Hamilton syringe, deposit $100 \mu \mathrm{l}$ lipid(s) solution onto roughened PTFE. Spread the solution on the surface to distribute it as evenly as possible.

3. Dry under vacuum $3 \mathrm{hr}$ to overnight at room temperature.

\section{Hydrate lipid film}

4. Prehydrate the resulting dry lipid film using moist nitrogen by bubbling nitrogen gas through sterile deionized water warmed on a hotplate and directing the resulting hydrated gas into a sealed chamber containing the lipid-coated PTFE sheet. Prehydrate film at least $30 \mathrm{~min}$.

If using light-sensitive membrane components, cover the chamber with aluminum foil.

5. Place PTFE sheet, lipid-side-up, on the bottom of the beaker. Fill with $5 \mathrm{ml}$ swelling solution. Cover beaker with Parafilm.

If using light-sensitive components, such as membrane dyes, wrap the beaker in aluminum foil.

6. Place beaker in incubator or oven and allow lipid film to swell into vesicles over 2 to 3 days.

The temperature during swelling should be higher than the chain-melting transition temperature of the highest-melting membrane component. For many unsaturated lipids, $35^{\circ}$ to $37^{\circ} \mathrm{C}$ gives satisfactory results.

The resulting suspension will be dense in giant vesicles of a variety of sizes and lamellarities. These can be transferred to sample chambers for microscope viewing or other experimentation using a pipet or syringe. Taking care to minimize shear stresses by using a large pipet-tip opening or syringe needle and slow extraction will maximize the number of vesicles that survive this transfer. If using membrane dyes, unilamellar vesicles can be identified under the microscope as vesicles with the lowest fluorescence from the membrane. 
PREPARING GUVS BY SWELLING OFF A UNIFORM FILM ON GLASS

This method requires more preparation time and more specialized equipment than Basic Protocol 1, but it produces a higher percentage of unilamellar vesicles in the final, giantvesicle suspension. This protocol also does not require charged lipids in the system to produce unilamellar vesicles if the swelling solution does not contain salt.

\section{Materials}

$80 \mu \mathrm{l}$ lipids in chloroform (Support Protocol 1) at $10 \mathrm{mM},(\sim 10 \mathrm{mg} / \mathrm{ml})$

Chloroform

Nitrogen gas

Swelling solution: $100 \mathrm{mM}$ sucrose in water (or other aqueous swelling solution), prepared using sterile deionized $\mathrm{H}_{2} \mathrm{O}$

Microdispensers with glass bores (Drummond), or Hamilton syringes

50- to 100 -ml glass flask with pointed bottom

Rotary evaporator

Vacuum oven

Oven or incubator

\section{Prepare dry lipid film in glass flask}

1. If using more than one species of lipid, mix in the desired ratio as in Support Protocol 1. Add membrane dye(s) and other components to be incorporated into the membrane, if desired, so that the total concentration of membrane components in chloroform solution remains $\sim 10 \mathrm{mM}$.

2. Using a glass microdispenser or Hamilton syringe, deposit $80 \mu \mathrm{l}$ of this solution in a 50- to 100-ml pointed-bottom glass flask.

3. Attach flask to a rotary evaporator in a near-horizontal orientation.

4. Evaporate while rotating flask in a water bath that is at a temperature greater than the chain-melting temperature of the highest-melting component in the system (for most unsaturated lipids and for saturated lipids with tails up to 16 carbon atoms long, $50^{\circ} \mathrm{C}$ is sufficient).

The resulting lipid film should be dry to the eye, with no remaining fluid discernible.

5. Dry this film for at least $2 \mathrm{hr}$ in a vacuum oven at a temperature greater than the chain-melting temperature of the highest-melting component in the system.

\section{Hydrate lipid film}

6. Pre-hydrate the resulting dry lipid film using warm, moist nitrogen by bubbling nitrogen gas through sterile deionized water warmed on a hotplate and directing the resulting hydrated gas into a sealed chamber containing the lipid-coated glass flask. Prehydrate film at least $30 \mathrm{~min}$.

If using light-sensitive membrane components, cover the chamber with aluminum foil.

7. Fill flask with sufficient swelling solution to cover the lipid film. Seal top with Parafilm.

If using light-sensitive components, cover flask with aluminum foil.

8. Incubate (in an oven or incubator, or in a water bath warmed on a hotplate) for 18 to $24 \mathrm{hr}$ at a temperature greater than the chain-melting temperature of the highestmelting component in the system.

Preventing vibrations and other mechanical disturbances during incubation is essential to a good yield of GUVs.
Lipids

24.3.9 
ALTERNATE PROTOCOL 3
If using membrane dyes, a successful incubation will be identified by a "cloud" of color in the approximate center of the vial.

9. Harvest vesicles from the center of the vial using a pipet or syringe, taking care to minimize shear forces, and transfer to sample chambers for microscope viewing or other experimentation.

As in Basic Protocol 1, unilamellar vesicles can be identified by low fluorescence from membrane dyes.

\section{PREPARING GUVS BY DEHYDRATION AND REHYDRATION OF SUVS}

This protocol uses dehydration and rehydration of small unilamellar vesicles (SUVs) to produce GUVs that adhere to a glass coverslip. This allows continuous microscopic observation of a single GUV or group of GUVs added as active agents directly to the system under study. At least 10 to $20 \mathrm{~mol} \%$ PC lipid seems to be required to form vesicular structures using this protocol.

\section{Materials}

100 to $300 \mu \mathrm{l}$ of lipid solution in chloroform at $20 \mathrm{mg} / \mathrm{ml}$ (Support Protocol 1) $100 \mathrm{mM} \mathrm{NaCl}$ (prepared using sterile deionized $\mathrm{H}_{2} \mathrm{O}$ ) containing 1 vol\% glycerol $100 \mathrm{mM} \mathrm{NaCl}$ (prepared using sterile deionized $\mathrm{H}_{2} \mathrm{O}$ ), without glycerol

Hamilton syringe

Small glass vial

Desiccator chamber, attached to vacuum pump

Bath sonicator $(43 \mathrm{kHz}$; L\&R Ultrasonics Model T21, http://www.lrultrasonics.com)

Glass coverslips

\section{Prepare dried lipid film in a glass vial}

1. If using more than one species of lipid, mix in the desired ratio as in Support Protocol 1. Add membrane dye(s) and other components to be incorporated into the membrane, if desired, so that the total concentration of membrane components in chloroform solution remains $\sim 20 \mathrm{mg} / \mathrm{ml}$.

2. Deposit 100 to $300 \mu \mathrm{l}$ of lipid solution in a small glass vial, place vial in vacuum desiccator, and dry under vacuum for $3 \mathrm{hr}$ to overnight.

\section{Hydrate lipid film and sonicate to form SUVs}

3. Hydrate lipid film in vial with $100 \mathrm{mM} \mathrm{NaCl}$ containing 1 vol\% glycerol. Add enough of this aqueous solution to produce a lipid concentration of $1 \mathrm{mg} / \mathrm{ml}$.

If $300 \mu \mathrm{l}$ of chloroform solution at $20 \mathrm{mg} / \mathrm{ml}$ is dried onto the vial, $6 \mathrm{ml}$ of aqueous solution is added.

4. Cap the vial and seal with Parafilm. If using light-sensitive components, cover with aluminum foil. Refrigerate for 24 to $48 \mathrm{hr}$ at $4^{\circ} \mathrm{C}$.

The refrigeration step is necessary for vesicle formation.

5. Sonicate lipid suspension in vial for 5 to $10 \mathrm{~min}$ to make small unilamellar vesicles (SUVs).

\section{Dry SUVs and rehydrate}

6. Deposit $5 \mu$ l of SUV suspension onto glass cover slip, spreading as much as possible.

7. Let dry 30 to $60 \mathrm{~min}$, long enough so that the droplet appears like a solid "gel," but not so long that it is visibly dry and cracked.

If using light-sensitive components, sample should be shielded from light while it is drying. 
8. Rehydrate using $100 \mathrm{mM} \mathrm{NaCl}$ solution (without glycerol), adding at least enough to cover the initially-deposited droplet $(10 \mu \mathrm{l})$.

Excess rehydrating solution (at least up to $500 \mu l$ ) may be added if desired. Giant vesicles form within 10 min. As in Basic Protocol 1, unilamellar vesicles can be identified by low fluorescence from membrane dyes.

\section{COMMENTARY}

\section{Background Information}

Giant unilamellar vesicles (GUVs) are liposomes up to tens of microns in size, consisting of a single lipid bilayer membrane. Due to their large size, they are especially amenable to studies using fluorescence and light microscopy, and may also be manipulated for mechanical measurements with optical traps or micropipets. In addition to their use in encapsulation, GUVs have proven to be useful model systems for studying many cellular processes, including tubulation (Roux et al., 2002; Koster et al., 2003) and budding, and fusion (Holopainen et al., 2000; Staneva et al., 2004), as well as peptide insertion (Thoren et al., 2004). The introduction of enzymes or proteins can result in reorganization, leading to such diverse behavior as vesicle aggregation, fusion, and fission (Nurminen et al., 2001).

More recently, GUVs have gained prominence as a platform for studying lipid phase separation as a model for the lipid raft hypothesis (Korlach et al., 1999; Dietrich et al., 2001; Baumgart et al., 2003). According to the lipid raft hypothesis, in-plane heterogeneity within cell membranes, in the form of lipid rafts, may play a key role in protein sorting and cell signaling (Simons and Ikonen, 1997). GUVs composed of simple mixtures of lipids and cholesterol display a rich phase behavior similar to that of lipid mixtures extracted from cells (Dietrich et al., 2001). This phase separation yields coexisting lipid phases, which differ in composition, density, and local curvature. GUVs provide model systems for the controlled study of the effects of lipid composition and protein binding on lipid phase formation, bending, line tension, budding, and stability.

\section{Critical Parameters}

\section{Lipid film thickness and uniformity}

It is generally desirable to obtain unilamellar vesicles larger than $10 \mu \mathrm{m}$ in size. To ensure a high yield of unilamellar vesicles free from small vesicles, it is important to begin with a thin lipid film without much roughness or too many defects. The protocols described here use rapid spreading to obtain this thin film.
When viewed in reflection, the film should appear nearly uniform, with slight variations in color. Films that are too thick will appear dull, and nonuniform films will appear patchy.

A more controlled alternative to spreading is spin-coating; however, this requires access to a spin-coater. These are typically available in engineering facilities. The parameters for creating a film of optimal thickness are described by Estes and Mayer (2005); we have also used this technique with success.

\section{Troubleshooting}

The most common problem associated with making GUVs is contamination of lipid or sugar solutions. It is recommended that chloroform be divided into smaller bottles for use in solutions and in cleaning. Lipid solutions should be stored under nitrogen or argon gas at $-20^{\circ} \mathrm{C}$ or lower. Small volumes of lipid should be used for mixtures, so that they may be discarded in case of contamination. Be attentive of changes in color or texture of lipid mixtures, as these are signs of contamination. Sugar solutions should be filter-sterilized, aliquotted, and frozen. Sugar solutions in use may be refrigerated, but should be brought to temperature before use in rehydration.

\section{Anticipated Results}

The protocols described should yield large vesicles, without many small vesicles or debris. By incorporating a small (0.1 to $1 \mathrm{~mol} \%)$ amount of fluorescently labeled lipid, it is easy to check with microscopy that vesicles are unilamellar, since multilamellar vesicles appear brighter, as shown in Figure 24.3.3B. Vesicles can also be viewed either with phase contrast or DIC microscopy, as shown in Figure 24.3.3B.

\section{Time Considerations}

The time required depends on the protocol chosen. Forming GUVs using electroformation protocols requires a minimum of $2 \mathrm{hr}$. Gentle hydration protocols require 1 to 3 days. For all protocols, much of the time required is for drying or hydration of the lipid films. 

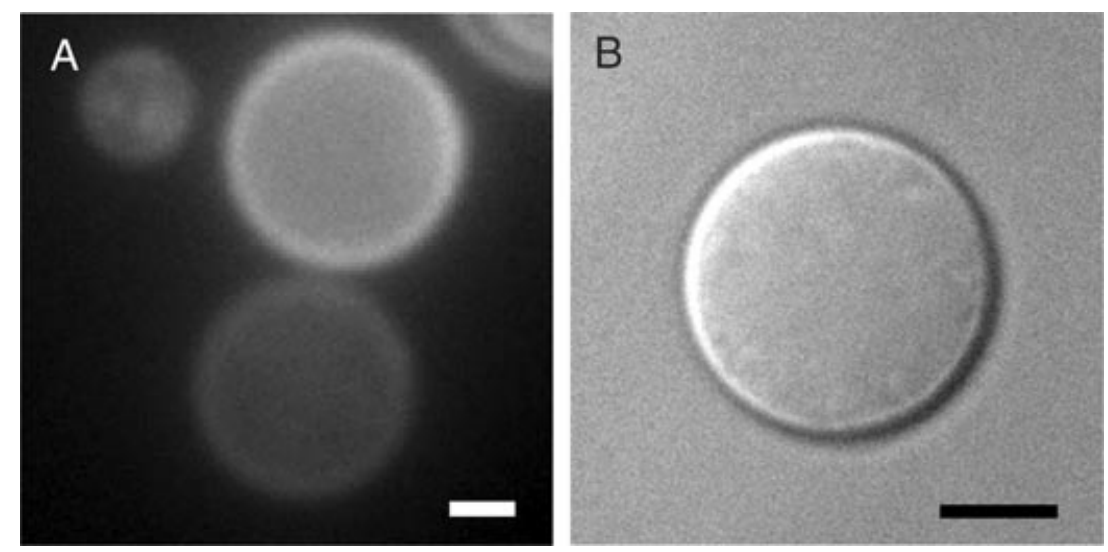

Figure 24.3.3 Giant vesicles, viewed with microscopy. (A) By incorporating $0.1 \mathrm{~mol} \%$ of a fluorescently labeled lipid, vesicles are readily viewed with fluorescence microscopy. Multilamellar vesicles appear brighter, as demonstrated by the upper vesicle. (B) Vesicles may also be viewed using phase microscopy. Scale bars $=10 \mu \mathrm{m}$.

\section{Literature Cited}

Angelova, M.I., Soleau, S., Meleard, P., Faucon, J.F., and Bothorel, P. 1992. Preparation of giant vesicles by external AC electric fields: Kinetics and applications. Progr. Colloid \& Polymer Sci. 89:127-131

Bacia, K., Schwille, P., and Kurzchalia, T.V. 2005. Sterol structure determines the separation of phases and the curvature of the liquid-ordered phase in model membranes. Proc. Natl. Acad. Sci. U.S.A. 102:3272-3277.

Bagatolli, L.A. 2006. To see or not to see: Lateral organization of biological membranes and fluorescence microscopy. Biochim. Biophys. Acta 1758:1541-1556.

Bagatolli, L.A. and Gratton, E. 2000. Two photon fluorescence microscopy of coexisting lipid domains in giant unilamellar vesicles of binary phospholipid mixtures. Biophys. J. 78:290-305.

Baumgart, T., Hess, S.T., and Webb, W.W. 2003. Imaging coexisting fluid domains in biomembrane models coupling curvature and line tension. Nature 425:821-824.

Beattie, M., Veatch, S.L., Stottrup, B., and Keller, S.L. 2005. Sterol structure determines miscibility vs. melting transitions in lipid vesicles. Biophys. J. 89:1760-1768.

Bigay, J., Gounon, P., Robineau, S., and Antonny, B. 2003. Lipid packing sensed by ArfGAP1 couples COPI coat disassembly to membrane bilayer curvature. Nature 426:563-566.

Dietrich, C., Bagatolli, L.A., Volovyk, Z.N., Thompson, N.L., Levi, M., Jacobson, K., and Gratton, E. 2001. Lipid rafts reconstituted in model membranes. Biophysical Journal 80:1417-1428.

Estes, D.J. and Mayer, M. 2005. Electroformation of giant liposomes from spin-coated films of lipids. Colloids and Surfaces B: Biointerfaces 42:115-123.

Gordon, V.D., Beales, P.A., Shearman, G.C., Zhao, Z., Seddon, J.M., Egelhaaf, S.U., and Poon, W.C.K. Solid-like domains in mixed lipid bilayers: Effects of lipid phase behavior, transition pathway, and membrane lamellarity. Manuscript in preparation.

Holopainen, J.M., Angelova, M.I., and Kinnunen, P.K.J. 2000. Vectorial budding of vesicles by asymmetrical enzymatic formation of ceramide in giant liposomes. Biophys. J. 78:830838.

Korlach, J., Schwille, P., Webb, W.W., and Feigenson, G.W. 1999. Characterization of lipid bilayer phases by confocal microscopy and fluorescence correlation spectroscopy. Proc. Natl. Acad. Sci. U.S.A. 96:8461-8466.

Koster, G., VanDujin, M., Hofs, B., and Dogterom, M. 2003. Membrane tube formation from giant vesicles by dynamic association of motor proteins. Proc. Natl. Acad. Sci. U.S.A. 100:1558315588.

Moscho, A., Orwar, O., Chiu, D.T., Biren, M.P., and Zare, R.N. 1996. Rapid preparation of giant unilamellar vesicles. Proc. Natl. Acad. Sci. U.S.A. 93:11443-11447.

Mueller, P., Chien, T.F., and Rudy, B. 1983. Formation and properties of cell-size lipid bilayer vesicles. Biophys. J. 44:375-381.

Nurminen, T.A., Holopainen, J.M., Zhao, H., and Kinnunen, P.K.J. 2001. Observation of topical catalysis by sphingomyelinase coupled to microspheres. J. Amer. Chem. Soc. 124:1212912134.

Roux, A., Cappello, G., Cartaud, J., Prost, J., Goud, B., and Bassereau, P. 2002. A minimal system allowing tubulation with molecular motors pulling 
on giant liposomes. Proc. Natl. Acad. Sci. U.S.A. 99:5394-5399.

Simons, K. and Ikonen, E. 1997. Functional rafts in cell membranes. Nature 387:569-572.

Staneva, G., Angelova, M.I., and Koumanov, K. 2004. Phospholipase A2 promotes raft budding and fission from giant liposomes. Chem. Phys. Lipids 129:53-62.

Thoren, P.E.G., Persson, D., Esbjorner, E.K., Goksor, M., Lincoln, P., and Norden, B. 2004. Membrane binding and translocation of cellpenetrating peptides. Biochemistry 43:34713489.

Veatch, S.L. and Keller, S.L. 2002. Organization in lipid membranes containing cholesterol. Phys. Rev. Lett. 89:268101.

Veatch, S.L. and Keller, S.L. 2003. Separation of liquid phases in giant vesicles of ternary mixtures of phospholipids and cholesterol. Biophys. J. 85:3074-3083. 